\title{
CHAOTIC VIBRATION OF AN AUTOPARAMETRICAL SYSTEM WITH THE SPHERICAL PENDULUM ${ }^{1}$
}

\author{
Danuta Sado, Jan Freundlich, Anna Bobrowska \\ Warsaw University of Technology, Institute of Machine Design Fundamentals, Warsaw, Poland \\ e-mail:dsado@poczta.onet.pl
}

\begin{abstract}
In the paper, the dynamics of a three degree of freedom vibratory system with a spherical pendulum in the neighbourhood of internal and external resonance is considered. It has been assumed that the spherical pendulum is suspended to the main body which is then suspended to the element characterized by some elasticity and damping. The system is excited harmonically in the vertical direction. The equation of motion has been solved numerically. The influence of initial conditions on the behaviour of the spherical pendulum is investigated. In this type of the system, one mode of vibration may excite or damp another one, and for different kinds of periodic vibrations there may also appear chaotic vibrations. For characterization of an irregular chaotic response, time histories, bifurcation diagrams, power spectral densities, Poincaré maps and the maximum Lyapunov exponents have been calculated.
\end{abstract}

Keywords: spherical pendulum, energy transfer, coupled oscillators, chaos

\section{Introduction}

The subject of this work is investigation of the effect of initial conditions on the dynamics of a three degree of freedom system with a spherical pendulum. Dynamical systems having an element in the form of a mathematical or physical pendulum have important applications. Different kinds of coupled autoparametric oscillators with simple pendulums are presented in the book by Sado (2010). Furthermore, the numerical and analytical methods of researching the dynamics of two degree and three degree of freedom vibratory systems with the pendulums are presented in this book. There are shown: the influence of initial conditions on the behaviour of the vibratory system, the characteristic of vibrations, and how to manipulate the vibrations.

The real pendulum is of spherical type. The spherical pendulum was investigated by many researches. The spherical pendulum subjected to parametric excitation was studied by Miles and Zou (1993). They investigated the non-linear response of a slightly detuned spherical pendulum with natural frequencies. In that model, no stable harmonic motion was possible, therefore the motion of the system was either periodically modulated by a sinusoid or becomes chaotic. The numerical integration was supported by analytical predictions, which also uncovered that the limit cycles and chaotic motions overlaped those of stable harmonic motion.

In paper by Naprstek and Fischer (2009), the pendulum vibration damper modelled as a two degree of freedom strongly non-linear auto-parametric system was investigated. There was a kinematic external excitation applied to a point. In that model the excitation was considered to be horizontal and harmonically variable in time. The solution and the stability were analysed. Therefore, the biggest attention was paid to the resonance domain. In certain domains of the pendulum and excitation parameters, the semi-trivial solution did not exist in that domain

\footnotetext{
${ }^{1}$ The paper was presented at the 27th Symposium on Vibrations in Physical Systems, May 9-13, Poznań-Będlewo, Poland.
} 
and various post-critical three-dimensional regimes occurred. Some of them were non-stationary despite the harmonic excitation. Three different types of the resonance domain were investigated. Their main properties depended on dynamic parameters of the pendulum and the external excitation amplitude. An analytical and numerical study brought some recommendations for designers of such devices. Their aim was to avoid any post-critical response rules risking the pendulum functionality.

The bifurcation behaviour of a spherical pendulum where the suspension point is harmonically excited in both vertical and horizontal directions was presented by Leung and Kung (2006). The equations of motion for a lightly damped spherical pendulum were considered. The point of the suspension was harmonically excited in both vertical and horizontal directions. The equations were solved with approximation in the neighbourhood of resonance by including the third order terms in the amplitude.

A mathematical model of the spherical pendulum with a moving pivot was suggested by Mitrev and Grigorov (2009) and later developed. Such a model allowed studying the influence of different kinematic excitations applied to the pivot point. That required the kinematical and dynamical parameters of the pendulum which also determined the force in the rope. A numerical simulation for spatial curvilinear and planar with straight line motions trajectories was performed. The stochastic analysis of a spring spherical pendulum was done by Viet (2015). The vibration was reduced by a spring and damper installed in the radial direction between the point mass and the cable. Under the sway motion, the centrifugal force resulted in the radial motion, which in its turn produced the Coriolis force to reduce the sway motion.

The dynamics of coupled spherical pendulums (where two lower pendulums were mounted at the end of the upper pendulum) was considered by Witkowski et al. (2014). The analysis showed that three rotating modes existed. The linear modes helped to understand the nonlinear normal modes, which were later visualized in plots. When there was an increase of energy in one mode, we could see a symmetry pitchfork bifurcation. In the second part of the paper, the energy transfer between pendulums was investigated. The results for co-rotating (all pendulums rotated in the same direction) and counter-rotating motion (one of lower pendulum rotated in the opposite direction) were presented.

In the present paper, it is assumed that the spherical pendulum is suspended on a flexible element, thus in this system there may occur an autoparametric excitation as a result of inertial coupling.

\section{A model of an autoparametric system with a spherical pendulum}

The investigated system is shown in Fig. 1 . The system consists of a body of mass $m_{1}$ suspended to the flexible element. This flexible element is characterised by the stiffness $k$ and damping $c$. This system also consists of the spherical pendulum of the length $l$ and mass $m_{2}$ which is suspended to the body of mass $m_{1}$. The body of mass $m_{1}$ is subjected to the harmonic vertical excitation $F_{1}(t)$ and the spherical pendulum is subjected to the horizontal excitation $F_{2}(t)$.

The spherical pendulum is similar to the simple pendulum but it moves in 3D space, so we need to introduce a new variable $\varphi$ in order to describe rotation of the pendulum in the plane $X Y$. The position of the body of mass $m_{1}$ is described only by the coordinate $z$. However, the position of the pendulum is described by the coordinate $z$ and two angles: $\theta$ and $\varphi$. The angle $\theta$ is the deflection of the pendulum measured from the vertical line. This system has three degrees of freedom. The equations of motion are derived as Lagrange's equations.

The kinetic energy $T$ is the sum of the energy of the two bodies

$$
T=\frac{m_{1} v_{1}^{2}}{2}+\frac{m_{2} v_{2}^{2}}{2}=\frac{m_{1} \dot{z}_{1}^{2}}{2}+\frac{m_{2}\left(\dot{x}_{2}^{2}+\dot{y}_{2}^{2}+\dot{z}_{2}^{2}\right)}{2}
$$




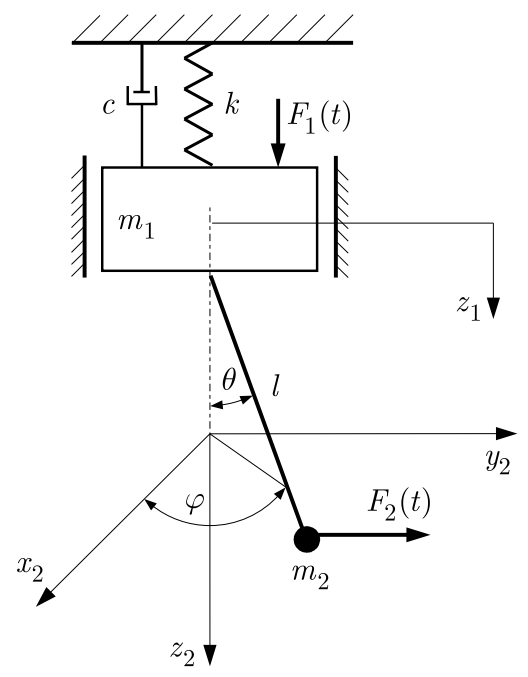

Fig. 1. A model of an autoparametric system with a spherical pendulum

where

$$
\begin{array}{lrl}
x_{2}=l \sin \theta \cos \varphi & y_{2}=l \sin \theta \sin \varphi & z_{2}=z_{1}+l \cos \theta \\
z_{1}=z+z_{s t} & z_{s t}=\frac{\left(m_{1}+m_{2}\right) g}{k}
\end{array}
$$

The kinetic energy $T$ is given by the expression

$$
T=\frac{1}{2}\left(m_{1}+m_{2}\right) \dot{z}^{2}+\frac{1}{2} m_{2}\left(l \dot{\theta}^{2}+l^{2} \dot{\varphi}^{2} \sin ^{2} \theta-2 l \dot{z} \dot{\theta} \sin \theta\right)
$$

The potential energy $V$ is given by the expression

$$
V=-\left(m_{1}+m_{2}\right) g\left(z+z_{s t}\right)+m_{2} g(l-l \cos \theta)+\frac{k\left(z+z_{s t}\right)^{2}}{2}
$$

Assuming that the exciting forces are in the form: $F_{1}(t)=P_{1} \cos \left(\nu_{1} t\right), F_{2}(t)=P_{2} \cos \left(\nu_{2} t\right)$, the dissipation function $D=c \dot{z}^{2} / 2$, the equations of motion of the system are as follows

$$
\begin{aligned}
& \left(m_{1}+m_{2}\right) \ddot{z}-m_{2} l \ddot{\theta} \sin \theta-m_{2} l \dot{\theta}^{2} \cos \theta+k z+c \dot{z}=P_{1} \cos \left(\nu_{1} t\right) \\
& m_{2} l^{2} \ddot{\theta}-m_{2} l \ddot{z} \sin \theta-m_{2} l^{2} \dot{\varphi}^{2} \sin \theta \cos \theta+m_{2} g l \sin \theta=l \cos \theta \sin \varphi P_{2} \cos \left(\nu_{2} t\right) \\
& m_{2} l^{2} \ddot{\varphi} \sin ^{2} \theta+2 m_{2} l^{2} \dot{\varphi} \dot{\theta} \sin \theta \cos \theta=l \sin \theta \cos \theta P_{2} \cos \left(\nu_{2} t\right)
\end{aligned}
$$

By introducing the dimensionless time and parameters

$$
\begin{array}{llll}
\tau=\omega_{1} t & \omega_{1}^{2}=\frac{k}{m_{1}+m_{2}} & \omega_{2}^{2}=\frac{g}{l} & \beta=\frac{\omega_{2}}{\omega_{1}} \\
\gamma=\frac{c}{\left(m_{1}+m_{2}\right) \omega_{1}} & \bar{z}=\frac{z}{l} & a=\frac{m_{2}}{m_{1}+m_{2}} & \mu_{2}=\frac{\nu_{2}}{\omega_{1}} \\
A_{1}=\frac{P_{1}}{\left(m_{1}+m_{2}\right) \omega_{1}^{2}} & A_{2}=\frac{P_{2}}{m_{2} l \omega_{1}^{2}} & \mu_{1}=\frac{\nu_{1}}{\omega_{1}} &
\end{array}
$$

after transformation (2.5) into the dimensionless form we obtain

$$
\begin{aligned}
& \ddot{z}-a \ddot{\theta} \sin \theta-\dot{\theta}^{2} \cos \theta+z+\gamma \dot{z}=A_{1} \cos \left(\mu_{1} \tau\right) \\
& \ddot{\theta}-\ddot{z} \sin \theta-\dot{\varphi}^{2} \sin \theta \cos \theta+\beta^{2} \sin \theta=A_{2} \cos \theta \sin \varphi \cos \left(\mu_{2} \tau\right) \\
& \ddot{\varphi} \sin \theta+2 \dot{\varphi} \dot{\theta} \cos \theta=A_{2} \cos \theta \cos \left(\mu_{2} \tau\right)
\end{aligned}
$$

where the overbars denoting non-dimensionalisation are omitted for convenience. 
After transformation, the equations of motion can be written in a simpler form for numerical calculations:

$$
\begin{aligned}
\ddot{z}= & \frac{1}{1-a \sin ^{2} \theta}\left[\left(A_{1} \cos \left(\mu_{1} \tau\right)+\dot{\theta}^{2} \cos \theta-z-\gamma \dot{z}\right)+a \sin \theta\left(\dot{\varphi}^{2} \sin \theta \cos \theta-\beta^{2} \sin \theta\right)\right] \\
\ddot{\theta}= & \frac{1}{1-a \sin ^{2} \theta}\left\{\dot{\varphi}^{2} \sin \theta \cos \theta-\beta^{2} \sin \theta\right. \\
& \left.+\left[A_{1} \cos \left(\mu_{1} \tau\right)+a \dot{\theta}^{2} \cos \theta-z-\gamma \dot{z}+a \sin \theta\left(\dot{\varphi}^{2} \sin \theta \cos \theta-\beta^{2} \sin \theta\right)\right] \sin \theta\right\} \\
\ddot{\varphi}= & \frac{1}{\sin ^{2} \theta}\left(A_{2} \sin \theta \cos \varphi \cos \left(\mu_{2} \tau\right)-2 \dot{\varphi} \dot{\theta} \sin \theta \cos \theta\right)
\end{aligned}
$$

\section{Numerical simulation results}

The equations of motion of the given model are solved numerically using Runge-Kutta method with a variable step length. The calculations are carried out for different values of parameters of the system and for different initial conditions. Exemplary time histories of the displacements $z$ and $\theta$ are obtained for parameters of the system $a=0.8, \beta=0.5, \gamma=0, A_{1}=A_{2}=0$ and for the initial conditions: $z(0)=0.1, \theta(0)=0.005^{\circ}, \varphi(0)=0, \dot{z}(0)=\dot{\theta}(0)=\dot{\varphi}(0)=0$ are presented in Fig. 2, where we can observe the energy transfer between the modes of vibration in a closed cycle. In this case, the spherical pendulum behaves in the same way as a simple pendulum, and motion of the pendulum is in the vertical plane (angle $\varphi$ is constant and it depends on the initial conditions, so it is equal to 0 for $\varphi(0)=0)$.

(a)

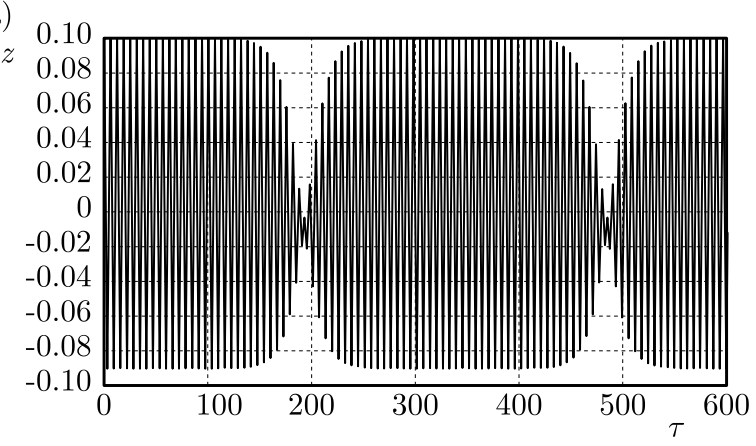

(b)

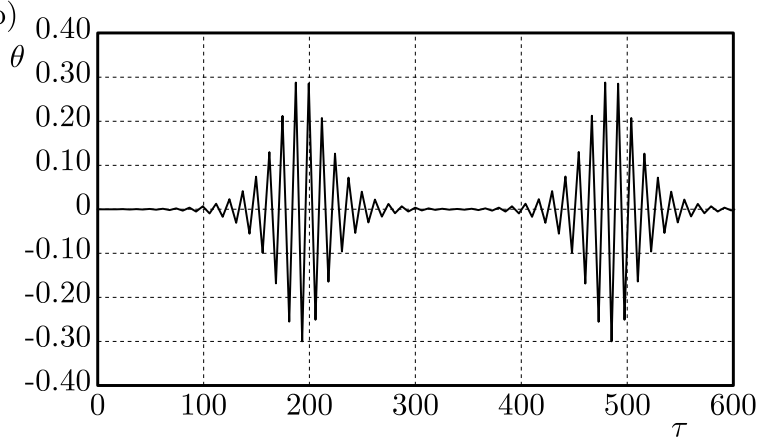

Fig. 2. Time history for (a) oscillator, (b) pendulum for $a=0.8, \beta=0.5, \gamma=0, A_{1}=A_{2}=0$, $z(0)=0.1, \theta(0)=0.005^{\circ}, \varphi(0)=0$

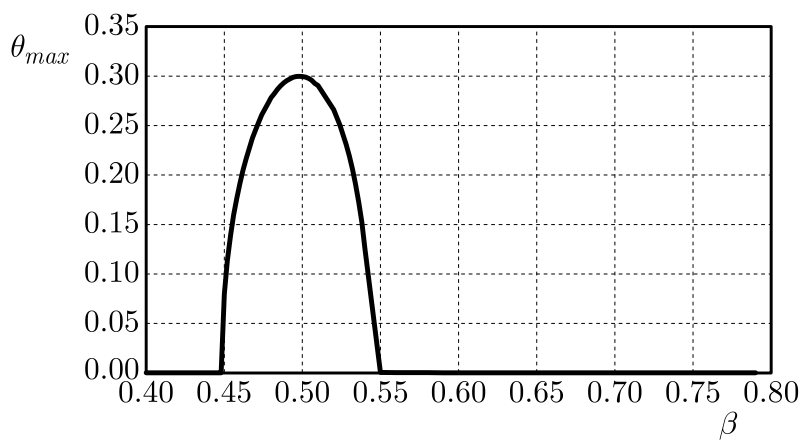

Fig. 3. Internal resonance for: $a=0.8, \gamma=0, A_{1}=A_{2}=0, z(0)=0.1, \theta(0)=0.005^{\circ}, \varphi(0)=0$

The diagram of internal resonance for the same initial conditions put on the displacements is presented in Fig. 3, and it is similar to a simple pendulum presented in the work (Sado, 2010). 
We observe the resonance excitation for the frequency ratio $\beta=0.5$. In this case, there is an assumption that the simple pendulum results are good.

On the other hand, when the initial conditions are put on the displacements and also on the velocities (in this example: $z(0)=0, \dot{z}(0)=0, \theta(0)=5^{\circ}, \dot{\theta}(0)=-0.04, \varphi(0)=0, \dot{\varphi}(0)=-0.96$ ), we can observe the influence of angle $\varphi$ (Fig. 4). For these initial conditions, we observe the oscillation displacements $z$ and $\theta$ with the same frequency.
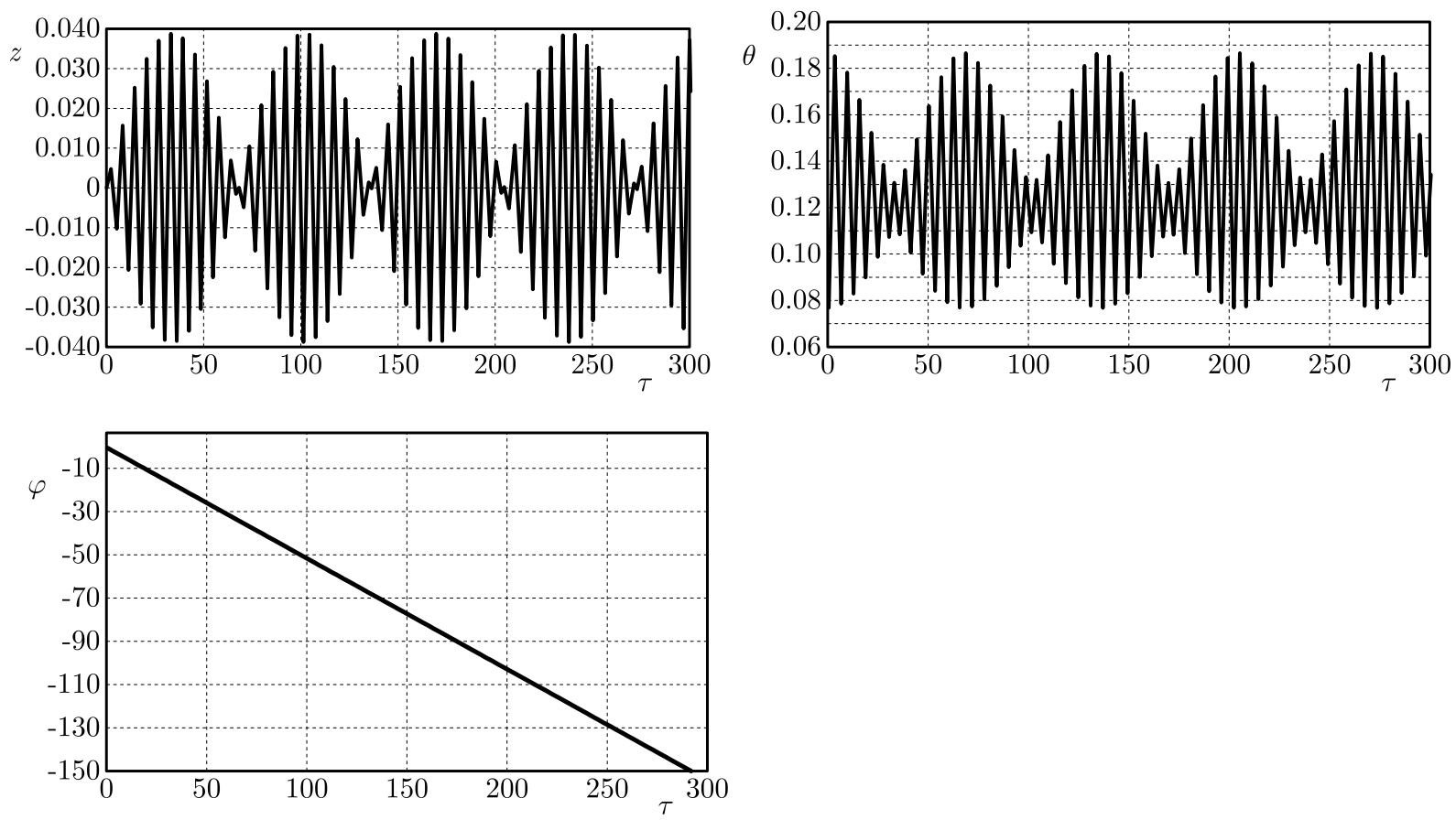

Fig. 4. Time history for: $a=0.5, \beta=0.51, \gamma=0, A_{1}=A_{2}=0, z(0)=0, \dot{z}(0)=0, \theta(0)=5^{\circ}$, $\dot{\theta}(0)=-0.04, \varphi(0)=0, \dot{\varphi}(0)=-0.96$

With the same initial conditions for displacements and velocities, the internal resonance is observed for the frequency ratio $\beta=0.51$ (shown in Fig. 5a).

(a)

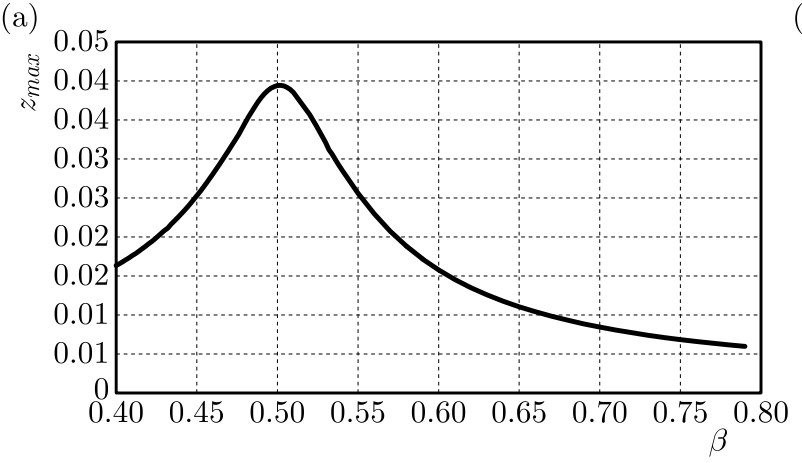

(b)

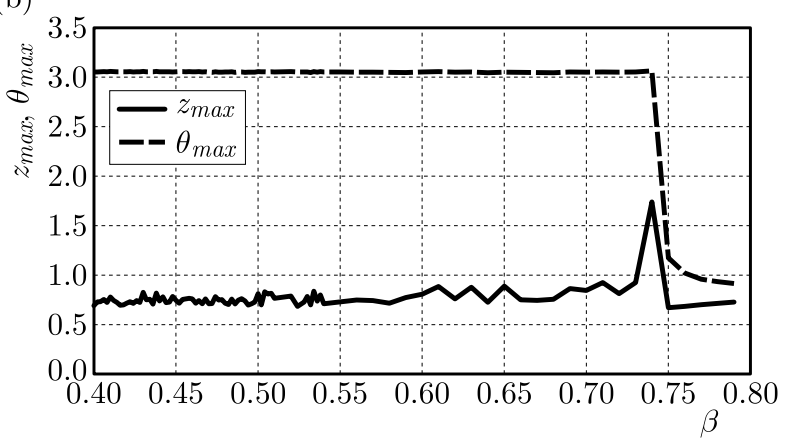

Fig. 5. Internal resonance for: (a) $a=0.5, \gamma=0, A_{1}=A_{2}=0, z(0)=0, \dot{z}(0)=0, \theta(0)=5^{\circ}$, $\dot{\theta}(0)=-0.04, \varphi(0)=0, \dot{\varphi}(0)=-0.96$; (b) $a=0.2, \gamma=0, A_{1}=A_{2}=0, z(0)=0, \dot{z}(0)=0.65$, $\theta(0)=50^{\circ}, \dot{\theta}(0)=-0.04, \varphi(0)=0, \dot{\varphi}(0)=-0.296$

For different parameters of the system: $a=0.2, \gamma=0, A_{1}=A_{2}=0, \beta=0.75$ and for initial conditions which are put on the displacements and also on the velocities $(z(0)=0, \dot{z}(0)=0.65$, $\left.\theta(0)=50^{\circ}, \dot{\theta}(0)=-0.04, \varphi(0)=0, \dot{\varphi}(0)=-0.296\right)$ there is visible an influence of the angle $\varphi$, and the internal resonance area is observed for the frequency ratio near $\beta=0.75$ (Fig. $5 \mathrm{~b}$ ). In this example, the angle $\varphi$ describes rotation of the pendulum around the axis $z$. 
Assuming the model of the pendulum as a spherical pendulum, we obtain results more similar to the real system.

Next we prepare diagrams of the external resonance. An example is considered when the initial conditions are imposed on the displacements: $a=0.8, \beta=0.5, \gamma=0, A_{1}=0.001$, $A_{2}=0, z(0)=0, \theta(0)=0.005^{\circ}, \varphi(0)=0$. In that case, the diagram shows the external resonance for both the coordinate $z$ and the angle $\theta$ with the vertical excitation force $F_{1}(t)$.
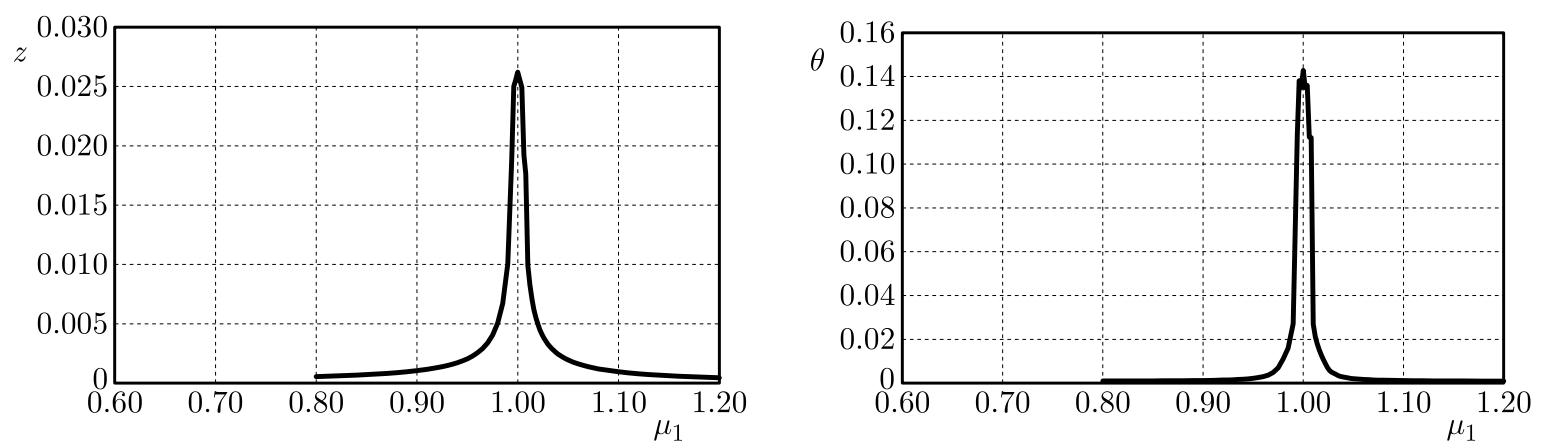

Fig. 6. External resonance for the coordinates $z$ and $\theta$ for: $a=0.2, \beta=0.5, \gamma=0, A_{1}=0.0001$, $z(0)=0, \gamma=0$

Near the internal and external resonances depending on the selection of physical parameters, the amplitudes of vibrations of the coupled system may be related differently. The motion in terms of $z$ and $\theta$ are periodic or quasi-periodic vibrations, but sometimes the motion of the body of mass $m_{1}$ and the pendulum are chaotic. To characterize an irregular chaotic response, bifurcation diagrams are constructed.

Exemplary bifurcation diagrams for the displacements $z$ and $\theta$ versus bifurcation parameter $A_{1}$ with parameters of the system: $a=0.2, \beta=0.5, \gamma=0.00081443, \mu=0.99$ are presented in Fig. 7 (it is assumed that the bifurcation parameter is the amplitude of excitation $A_{1}$ ).
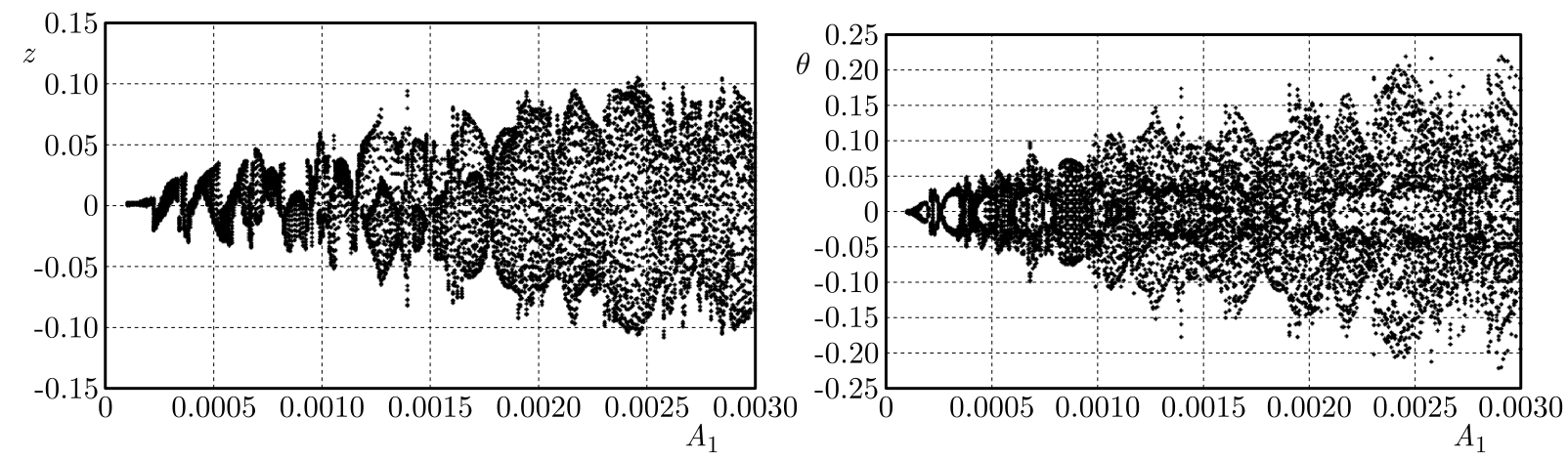

Fig. 7. Bifurcation diagrams for the coordinates $z$ and $\theta$ for: $a=0.2, \beta=0.5, \gamma=0.00081443, \mu_{1}=0.99$

As it can be seen from the bifurcation diagrams, motion of the oscillator and the spherical pendulum depends of the amplitude of excitation $A_{1}$ and can be of different character: it maybe periodic, quasi-periodic or chaotic. Several phenomena can be observed: the existence of a simple or a chaotic atractor, and various bifurcations. All these phenomena have to be verified in the phase space. Therefore, the Poincaré maps and Lyapunov exponents are then constructed. Exemplary results of the Poincaré maps (Fig. 8) and the maximum Lyapunov exponents (Fig. 9) are presented for the amplitude of excitation $A_{1}=0.0029$.

It is visible that the Poincaré maps trace strange atractors and the maximum Lyapunov exponents are positive, so the motions with respect both coordinates are chaotic. 

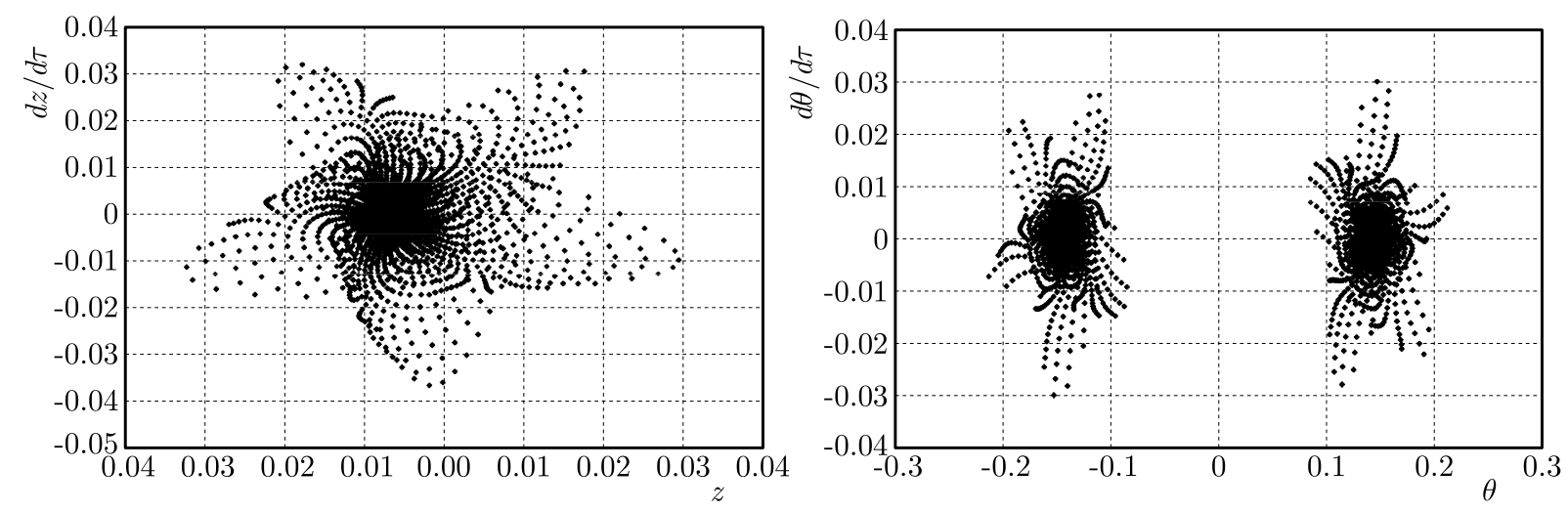

Fig. 8. Poincaré maps for the coordinates $z$ (a) and $\theta$ (b) for $a=0.2, \beta=0.5, \gamma=0.00081443$, $\mu_{1}=0.99, A_{1}=0.0029$

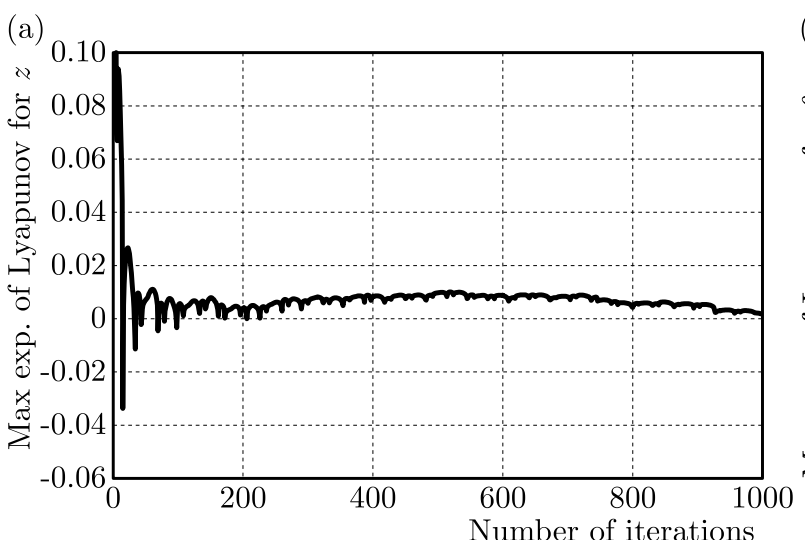

(b)

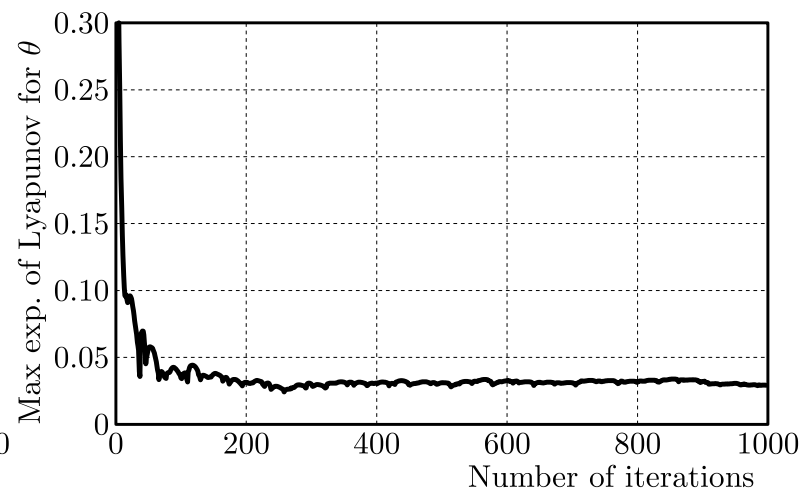

Fig. 9. Maximum Lyapunov exponent for the coordinates $z$ (a) and $\theta$ (b) for $a=0.2, \beta=0.5$, $\gamma=0.00081443, \mu_{1}=0.99, A_{1}=0.0029$

\section{Conclusions}

The influence of initial conditions on the behaviour of an autoparametric system with a spherical pendulum is very interesting, because sometimes when the initial conditions are imposed on the displacements of the spherical pendulum it behaves similarly to a simple pendulum (angle $\varphi$ is constant), but when the initial conditions are put also on the velocities, we observe some influence of the angle $\varphi$. It is important, because near the internal and external resonance area there may appear different motion - regular or chaotic. Autoparametric systems are very sensitive to nonlinearities and the energy is transferred between modes of vibrations in a closed cycle. The time of this cycle depends on the values of parameters, and numerical calculations should be carried for a satisfactorily long time. Assuming the model of the pendulum as the spherical pendulum, the obtained results are more similar to those obtained for the system with the simple pendulum.

\section{References}

1. Leung A.Y.T., KuAng J.L., 2006, On the chaotic dynamics of a spherical pendulum with a harmonically vibrating suspension, Nonlinear Dynamics, 43, 213-238

2. Miles J.W., Zou Q.P., 1993, Parametric excitation of a detuned spherical pendulum, Journal of Sound and Vibration, 164, 2, 237-250 
3. Mitrov R., Grigorov B., 2009, Dynamic behaviour of a spherical pendulum with spatially moving pivot, Zeszyty Naukowe Politechniki Poznańskiej, 9, 81-91

4. NAprstek J., Fischer C., 2009, Auto-parametric semi-trival and post-critical response of a spherical pendulum damper, Computers and Structures, 87, 1204-1215

5. SAdo D., 2010, Regular and Chaotic Vibration in Selected Pendulum Systems (in Polish), WNT, Warsaw

6. Viet L.D., 2015, Partial stochastic linearization of a spherical pendulum with Coriolis damping produced by radial spring and damper, Journal Vibration and Acoustics, 137, 5, 1-9, DOI: 10.1115/1.4030663

7. Witkowski B., Perlikowski P., Prasad A., Kapitaniak T., 2014, The dynamics of co- and counter rotating coupled spherical pendula, The European Physical Journal Special Topics, 223, 4, $707-720$

Manuscript received September 20, 2016; accepted for print January 16, 2017 